\title{
Flexibility of ED surveillance system to monitor dengue outbreak in Reunion Island
}

\section{Pascal Vilain ${ }^{1}$, Muriel Vincent ${ }^{1}$, Anne Fouillet ${ }^{2}$, Katia Mougin-Damour ${ }^{3}$, Xavier Combes $^{4}$, Adrien Vague $^{5}$, Fabien Vaniet ${ }^{5}$, Laurent Filleul ${ }^{2}$, Luce Menudier ${ }^{1}$}

${ }^{1}$ Regional office of French National Public Health Agency in Indian Ocean, Saint-Denis, Réunion, ${ }^{2}$ French National Public Health Agency, SaintMaurice, France, ${ }^{3}$ Hospital Centre, Saint-Paul, Réunion, ${ }^{4}$ University Hospital Centre, Saint-Denis, Réunion, ${ }^{5}$ Hospital Centre, Saint-Benoît, Réunion

\section{Objective}

To describe the characteristics of ED vitis related to dengue fever and to show how the syndromic surveillance system can be flexible for the monitoring of this outbreak.

\section{Introduction}

In Reunion Island, a French overseas territory located in the southwestern of Indian Ocean, the dengue virus circulation is sporadic. Since 2004, between 10 and 221 probable and confirmed autochthonous dengue fever cases have been reported annually. Since January 2018, the island has experienced a large epidemic of DENV serotype 2. As of 4 September 2018, 6,538 confirmed and probable autochthonous cases have been notified[1]. From the beginning of the epidemic, the regional office of National Public Health Agency (ANSP) in Indian Ocean enhanced the syndromic surveillance system in order to monitor the outbreak and to provide hospital morbidity data to public health authorities.

\section{Methods}

In Reunion Island, the syndromic surveillance system called OSCOUR ${ }^{\circledR}$ network (Organisation de la Surveillance Coordonnée des Urgences) is based on all emergency departments (ED) [2]. Anonymous data are collected daily directly from the patients' computerized medical files completed during medical consultations. Every day, data files are sent to the ANSP via a regional server over the internet using a file transfer protocol. Each file transmitted to ANSP includes all patient visits to the ED logged during the previous 24 hours (midnight to midnight). Finally, data are integrated in a national database (including control of data quality regarding authorized thesauri) and are made available to the regional office through an online application [3].

Following the start of dengue outbreak in week 4 of 2018, the regional office organized meetings with physicians in each ED to present the dengue epidemiological update and to recommend the coding of ED visit related to dengue for any suspect case (acute fever disease and two or more of the following signs or symptoms: nausea, vomiting, rash, headache, retro-orbital pain, myalgia). During these meetings, it was found that the version of ICD-10 (International Classification of Diseases) was different from one ED to another. Indeed, some ED used A90, A91 (ICD-10 version: 2015) for visit related to dengue and others used A97 and subdivisions (ICD-10 version: 2016). As the ICD-10 version: 2015 was implemented at the national server, some passages could be excluded. In this context, the thesaurus of medical diagnosis implemented in the national database has been updated so that all codes can be accepted. ED visits related to dengue fever has been then described according to age group, gender and hospitalization.

\section{Results}

From week 9 of 2018, the syndromic surveillance system was operational to monitor dengue outbreak. The regional office has provided each week, an epidemic curve of ED visits for dengue and a dashboard on descriptive characteristic of these visits. In total, $441 \mathrm{ED}$ visits for dengue were identified from week 9 to week 34 of 2018 (Figure 1). On this period, the weekly number of ED visits for dengue was correlated with the weekly number of probable and confirmed autochthonous cases $($ rho $=0.86$, $p<0.001)$. Among these visits, the male/female ratio was 0.92 and median (min-max) age was 44 (2-98) years. The distribution by age group showed that 15-64 year-old $(72.1 \%, \mathrm{n}=127)$ were most affected. Age groups 65 years and more and 0-14 year-old represented respectively $21.8 \%(\mathrm{n}=96)$ and $6.1 \%(\mathrm{n}=27)$ of dengue visits. About $30 \%$ of dengue visits were hospitalized.

\section{Conclusions}

According Buehler et al., "the flexibility of a surveillance system refers to the system's ability to change as needs change. The adaptation to changing detection needs or operating conditions should occur with minimal additional time, personnel, or other

ISDS Annual Conference Proceedings 2019. This is an Open Access article distributed under the terms of the Creative Commons AttributionNoncommercial 4.0 Unported License (http://creativecommons.org/licenses/by-nc/3.0/), permitting all non-commercial use, distribution, and reproduction in any medium, provided the original work is properly cited. 
ISDS 2019 Conference Abstracts

resources. Flexibility generally improves the more data processing is handled centrally rather than distributed to individual dataproviding facilities because fewer system and operator behavior changes are needed..." [4]. During this dengue outbreak, the syndromic surveillance system seems to have met this purpose. In four weeks (from week 5 to week 9 of 2018), the system was able to adapt to the epidemiological situation with minimal additional resources and personnel. Indeed, updates were not made in the IT systems of each EDs' but at the level of the national ANSP server (by one person). This surveillance system was also flexible thank to the reactivity of ED physicians who timely implemented coding of visits related to dengue fever.

In conclusion, ED surveillance system constitutes an added-value for the dengue outbreak monitoring in Reunion Island. The automated collection and analysis data allowed to provide hospital morbidity (severe dengue) data to public health authorities. Although the epidemic has decreased, this system also allows to continue a routine active surveillance in order to quickly identify a new increase.

\section{Acknowledgement}

All emergency departments of the Reunion Island.

\section{References}

1. Santé publique France. Surveillance de la dengue à la Réunion. Point épidémiologique au 4 septembre 2018. http://invs.santepubliquefrance.fr/fr/Publications-et-outils/Points-epidemiologiques/Tous-les-numeros/OceanIndien/2018/Surveillance-de-la-dengue-a-la-Reunion.- Point-epidemiologique-au-4-septembre-2018. [Accessed September $8,2018]$.

2. Vilain P, Filleul F. La surveillance syndromique à la Réunion: un système de surveillance intégré. [Syndromic surveillance in Reunion Island: integrated surveillance system]. Bulletin de Veille Sanitaire. 2013;(21):9-12. http://invs.santepubliquefrance.fr/fr/Publications-et-outils/Bulletin-de-veille-sanitaire/Tous-les- numeros/Ocean-indienReunion-Mayotte/Bulletin-de-veille-sanitaire-ocean-Indien.-N-21-Septembre-2013. [Accessed September 4, 2018].

3. Fouillet A, Fournet N, Caillère N, et al. 2013. SurSaUD® Software: A Tool to Support the Data Management, the Analysis and the Dissemination of Results from the French Syndromic Surveillance System. OJPHI. 5(1), e118. https://doi.org/10.5210/ojphi.v5i1.4426

4. Buehler JW, Hopkins RS, Overhage JM, Sosin DM, Tong V, \& CDC Working Group. 2004. Framework for evaluating public health surveillance systems for early detection of outbreaks: recommendations from the CDC Working Group. MMWR Recomm Rep. 53(RR-5), 1-11. PubMed 


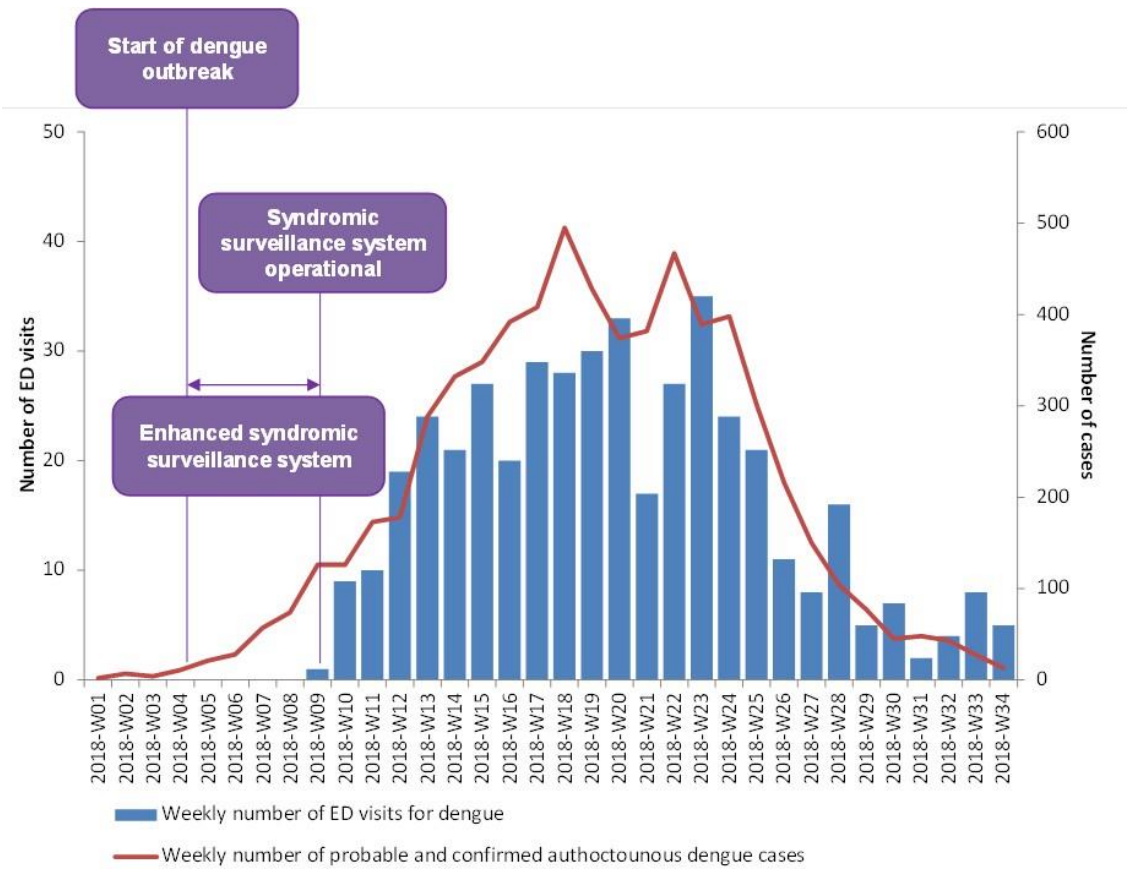

Figure 1. Weekly number of ED visits related to dengue and weekly number of probable and confirmed autochthonous dengue fever cases, from week 1 to week 34, 2018, Reunion Island. 